\title{
Cytokine Storm of SARS-CoV-2, the Virus that Causes COVID-19
}

\author{
Hassan Naji
}

\begin{abstract}
The ongoing pandemic of COVID-19 has spread to the entire globe and is a public health emergency of international concern. The clinical picture of the disease is seen to be related to previously encountered beta-coronaviruses i.e. SARS-CoV and MERS-CoV. Most common symptoms include fever and cough and less commonly diarrhea, myalgias, nausea, sputum production. Still, other patients can present with multiorgan failure. Mortality risk is related to patient's age and comorbidities. Laboratory and radiologic investigations are crucial for diagnosis and monitoring the prognosis of patients. The pathophysiology of COVID-19 is related to pulmonary inflammation and lung damage caused by the cytokine storm induced by local infection in the lung alveolar space. The cytokine storm is evident from an increase in the amount of several cytokines like IL1B, IL6, IL7, IL8, IL9, IL10, TNF, an INF $\gamma$. At present, a proper treatment for COVID-19 is unavailable. However, supportive care is being given to patients with anti-viral, anti-inflammatory, anti-bacterial, anti-fungal and artificial oxygen respiration. This paper focuses on the cytokine storm observed following SARS-CoV-2 infection and the current treatment strategies for the infection.
\end{abstract}

Index Terms - SARS-CoV-2, 2019 Novel Coronavirus, Cytokine Storm, COVID-19, Treatment, ALT, AST, Interleukin, IL, Janus Kinase, Interferon, INF, Corticosteroids, Methylprednisolone, Methylprednisolone Sodium Succinate, Dexamethasone, Anakinra, Antiviral, Remdesivir, Oseltamivir, Ganciclovir, Ritonavir, Lopinavir, Cytokine Storm Syndrome (CSS)

\section{INTRODUCTION}

In December 2019, a series of unknown etiology of pneumonia cases was reported in Wuhan, Hubei province, China. This newly recognized illness rapidly spread throughout China to other countries worldwide. On January 7, 2020, the Chinese Center for Disease Control and Prevention (CDC) identified a novel coronavirus by taking the throat swab sample of an infected patient. The virus was initially named 2019 novel coronavirus (2019-nCoV) (Huang et al., 2020). On February 11, 2020, the World Health Organization (WHO) officially named this virus as SARSCoV-2 (Severe Acute Respiratory Syndrome Coronavirus 2) and declared the disease caused by SARS-CoV-2 (COVID19: coronavirus disease-19, the number 19 is related to the fact that the disease was first recognized in 2019) a public health emergency of international concern. As of February 5, 2020 , the total number of 24,554 laboratory confirmed cases of COVID-19 have been reported globally in over 60 countries including the USA (Guan et al., 2020).

Coronaviruses have known to infect humans since 1960s.
Coronaviruses are zoonotic viruses and have been identified in mammals, including bats, mice, civets, cats, dogs and in several avian hosts. They are enveloped viruses, composed of single-stranded, positive-sense RNA genome. Coronaviruses belong to Coronaviridae family in the order Nidovirales. The genome of coronavirus is the second largest genome among RNA viruses, reaching up to $30 \mathrm{~kb}$ in length, which provides it greater chances of genetic diversity and cross-species transmission (ICTV, 2018). In the past two decades, the two other beta-coronaviruses namely SARS-CoV (Severe Acute Respiratory Syndrome-coronavirus) and MERS-CoV (Middle East Respiratory Syndrome-coronavirus) caused epidemics that resulted in more than 10,000 infected cases. The mortality rate associated with SARS-CoV was $10 \%$ and with MERS-CoV was 37\% (Huang et al., 2020). The phylogenetic analysis of COVID-19 indicates that it is closer to two bat coronaviruses namely, bat-SL-CoVZXC21 and bat-SL-CoVZC45 at the whole genome level.

Infection mainly spreads through human to human transmission and is highly contagious. The mortality rate associated with SARS-CoV-2 infection is approximately $3.7 \%$ and the leading cause of mortality is respiratory failure due to acute respiratory distress syndrome (Mehta et al., 2020). The clinical features of the COVID-19 ranges from mild to severe in patients with an underlying chronic disease. Early clinical features include, fever, headache, nonproductive cough, diarrhea, myalgias, nausea, and vomiting. The laboratory findings of the patients revealed lymphopenia. thrombocytopenia, increased levels of liver enzymes, lactate dehydrogenase (LDH), C-reactive protein (CRP), creatine kinase, blood urea nitrogen, procalcitonin, creatinine, and an increase in the levels of several cytokines referred to as 'cytokine storm'. On chest CT, patients show ground-glass opacity and multiple infiltrates.

\section{CLINICAL MANIFESTATION OF COVID-19}

The clinical manifestations of COVID-19 vary among individuals resembling largely to those of SARS-CoV. The incubation period of COVID-19 ranges from 3 to 6 days (Chen et al., 2020). Most common symptoms include fever (mainly low fever), nonproductive cough, sore throat, myalgia and shortness of breath. Some patients can also present with headache, diarrhea, chest pain, sputum production and confusion, in the absence of fever and cough (Huang et al., 2020). Most patients developed fever after being hospitalized. Still other patients can present with organ dysfunction including acute respiratory distress syndrome, acute respiratory injury, septic shock, acute renal injury and 
ventilator-associated pneumonia. Among these, acute respiratory distress syndrome is the most frequently observed. Like MERS-CoV, patients with an underlying chronic disorder mainly diabetes, cardiovascular and cerebrovascular diseases are more susceptible to COVID-19 as compared to immunocompetent individuals. The differences in the severity of COVID-19 infection in different individuals is likely associated with host factors (Wang et al., 2020).

Studies shown that SARS-CoV-2 infects mostly men $(70 \%)$ than women (Yang et al., 2020). This variation is thought to be related to $\mathrm{X}$ chromosome and sex hormones of females that plays a vital role in both, innate and adaptive immune responses. SARS-CoV-2 infection in patients with compromised immune system such as, diabetics, older aged individuals, patients with AIDS, pregnant females, and individuals using immunosuppressive drugs can cause severe complications if not promptly treated with antibiotics. Some severely ill patients can also develop secondary bacterial and fungal infections by Klebsiella pneumoniae, Candida albicans, Candida glabrata, Aspergillus flavus, Aspergillus fumigatus, ESBL-negative Serratia marcescens, and drugresistant strains of Acinetobacter baumannii (Chen et al., 2020). The complications include acute respiratory distress syndrome, RNAaemia, acute cardiac injury, and secondary infections. The mortality rate is high in critically ill patients infected with SARS-CoV-2.

Radiologic confirmation of COVID-19 pneumonia can be made after 5 days from the onset of symptoms (Yang et al., 2020). The typical abnormalities in chest CT images are bilateral ground glass opacity, local or bilateral patchy shadows, bilateral multi-lobular, subsegmental areas of consolidation and interstitial abnormalities.

\section{Cytokine StORM OF COVID-19}

The complete blood count profile of individuals on presentation reveals leukopenia, lymphopenia, thrombocytopenia, neutrophilia, and decreased hemoglobin levels. However, some can also present with increased leukocytes (leukocytosis) and platelet counts (thrombocytosis). The coagulation profile of infected patients is also disturbed with increased prothrombin time (PT), Ddimer, and fibrinogen levels (Huang et al., 2020). Levels of liver function enzymes i.e. aspartate aminotransferase (AST) and alanine aminotransferase (ALT) are also deranged with the abnormality proportional to the degree of disease. Remaining abnormal blood biochemistry findings include decreased albumin and increased levels of total bilirubin, blood urea nitrogen (BUN), glucose, creatine kinase, serum creatinine, myoglobin, and lactate dehydrogenase. The infection related biomarkers are also raised including procalcitonin, serum ferritin, C-reactive protein, erythrocyte sedimentation rate (ESR) and interleukins. In addition, an elevated level of hypersensitive troponin-I can be observed in patients developing virus-related cardiac injury (Chen et al., 2020). Patients also have elevated soluble CD25 levels. Increased levels of a vast variety of pro-inflammatory cytokines can be found in the plasma of infected individuals such as IL6, IL7, IL8, IL9, IL10, IL1B, IL1RA, M1P1A, M1P1B, MCP1, IFN $\gamma$, GCSF, GMCSF, basic FGF, TNF $\alpha$, VEGF, and PDG indicating a cytokine storm (Figure 1).
SARS-CoV-2 infection can be confirmed by real-time RTPCR testing of respiratory tract specimens. About 26\% patients with COVID-19 become so sick by the cytokine storm that they require hospitalization and among them a significant number of patients require admission to the intensive care unit (Wang et al., 2020).

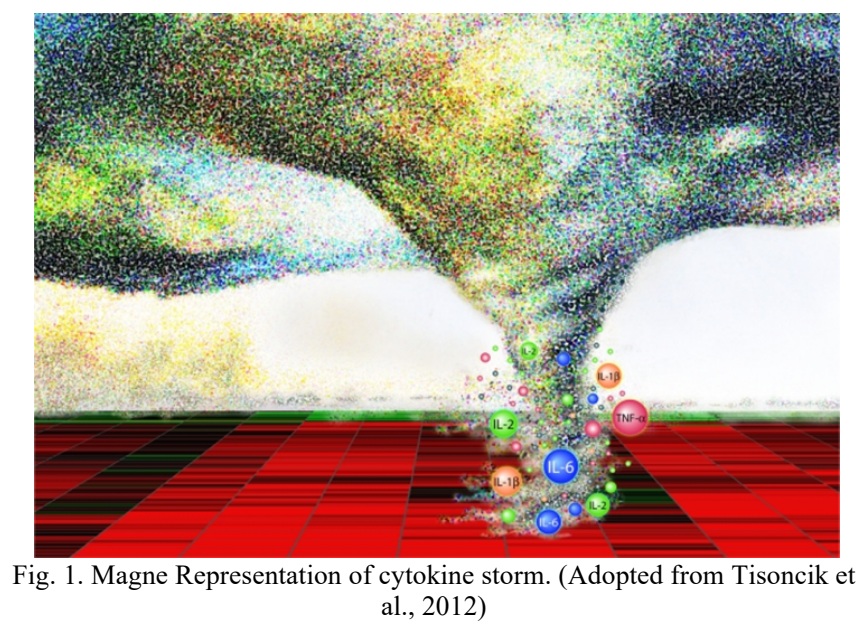

The elevated levels of various cytokines in COVID-19 demonstrates the cytokine storm syndrome (CSS), also seen with SARS-CoV and MERS-CoV infections. The elevated levels of ferritin, lymphocytes, lactate dehydrogenase (LDH), soluble CD25, prothrombin time, and several cytokines especially IL-6 in critically ill patients are suggestive of cytokine storm syndrome (CSS) in these patients. The cytokine storm syndrome (CSS) is most frequently observed in patients admitted in the intensive care unit. This CSS in COVID-19 patients is due to the result of hyperactive immune response because of infection. CSS is also seen in disorders like secondary hemophagocytic lymphohistiocytosis (sHLH) and macrophage activation syndrome (MAS). The COVID-19 predominantly attaches to angiotensin-converting enzyme 2 (ACE2) receptor in the lower respiratory tract. The similarities between the sequence of SARS-CoV-2 and SARS-CoV suggest that SARS-CoV-2 with the aid of ACE2 receptors may also infect lower airway ciliated epithelial cells (Wang et al., 2020). The levels of TNF, IL1B, IL-8, IL-6, and MCP1 are increased within minutes to hours following infection. However, the levels of other cytokines remain normal in the infected patients and these include RANTES, eotaxin, IL15, IL-5, and IL12p70. This cytokine storm is generated when virus particles infect and spread through the respiratory mucosa in the body. Increased levels of IL6, IL1B, IL12, and MCP1, leads to activated T-helper 1 (Th-1) cell responses. The activation of Th-1 cells generates a series of immune responses and changes the number of peripheral leukocytes. Despite the activation of Th-1 cells, the total lymphocyte count is decreased in patients, suggesting that SARS-CoV-2 mainly infects lymphocytes. This lymphocytopenia is related to the severity of SARS-CoV-2 infection (Chen et al., 2020). Similarly, the cytokine storm is also related to disease severity as evident by higher concentration in ICU patients as compared to non-ICU patients. The cytokine storm then spills over into the systematic circulation, causing leukopenia and thrombocytopenia. The cytokine storm induced by infection 
in lung alveolar environment can progress into acute respiratory distress syndrome or acute lung injury, as seen in various patients with SARS-CoV-2 infection, similar to SARS-CoV and MERS-CoV. Therefore, early diagnosis and treatment of critically ill patients is necessary for good prognosis. The key cytokine associated with lung injury is IL1B. The lung injury caused by cytokine storm syndrome can also progress to septic shock and multiple organ failure.

It is suggested that the level of IL- 6 could be used to predict the poor prognosis of COVID-19 patents (Xiaohua et al., 2020). The serum SARS-CoV-2 nucleic acid (RNAaemia) can be detected only in fraction of patients with COVID-19. Such incidence of correlation between RNAaemia and occurrence of cytokine storm is related to the level of IL-6. Patients who exhibited severe cytokine storms and required close monitoring is ICU have shown high level of IL-6. This has implication on the treatment and management of COVID19. Recent correlation between the serum viral load detection (RNAaemia) and the level of IL-6 were assessed and found to be highly correlated $(\mathrm{R}=0.902)$. On the other hand, patients with poor prognosis of COVID-19 and their level of IL-6 were correlated $(\mathrm{R}=0.682)$ indicating the potential usage of the level of IL-6 to predict the severity of COVID-19. Such association of level of IL-6 and cytokine storm have a potential usage in the treatment of critically ill patient with excessive targeted antiinflammatory drugs for IL-6 (Xiaohua et al., 2020).

In addition to the increased secretion of pro-inflammatory cytokines from the T-lymphocytes, SARS-CoV-2 also leads to increased secretion of anti-inflammatory cytokines including IL-4 and IL-10, from T-helper-2 (Th-2) cells. This finding is unique to SARS-CoV-2 as compared to SARS$\mathrm{CoV}$ infection. Studies have shown that genetic polymorphisms in Toll-like receptors (TLRs) are related to the severity of sepsis and cytokine storm in individuals as viral infection in the lungs induces inflammation through interaction with TLRs, NOD-like and RIG-I-like receptors (Tisoncik et al., 2012).

Similarly, mice models reported that mice with perforin deficiency died within two weeks of developing CSS after infected with lymphocyte choriomeningitis virus. Interestingly, mice with removed CD8 T-lymphocytes and with neutralized IFN $\gamma$ successfully survived the infection. It is evident that death of the mice was due to immune response to the virus instead of the viral load. Though people are at risk of developing SARS-CoV-2 infection, yet only a small percentage of individuals have genetic factors that contributes to frequently deadly CSS (Wang et al., 2020).

\section{TREATMENT}

Meantime, there is no effective and proper medication for the treatment of COVID-19 infection, so the focus is on providing supportive care to the patients. The goals of treatment for COVID-19 infection involve reduction of viral load and the widespread inflammation due to cytokine storm in the body, and alleviation of hypoxemia in the patients. The current treatment strategies are administration of anti-viral drugs, anti-inflammatory drugs, selective cytokines blockage, Janus Kinase (JAK) inhibition and providing oxygen support to patients in isolation. It is also recommended to use intravenous immunoglobulins in severely ill patients to provide passive immunity against infection because the COVID-19 decreases immune cells and inhibits the body's cellular immune functions that is evident by lymphopenia. It is suggested that the destruction of T-lymphocytes is the leading factor to the worsening of patients (Chen et al., 2020).

Anti-inflammatory therapy involves administration of systematic corticosteroids, both orally and intravenously. The recommended dose of corticosteroids is low to moderate. The recommended steroids include methylprednisolone, methylprednisolone sodium succinate, and dexamethasone. The use of methylprednisolone is recommended for patients with acute respiratory distress syndrome (ARDS), but for a short interval of treatment as possible usually 3-15 days. If the patient is also diagnosed with community-acquired pneumonia, methylprednisolone can also be administered as a combined regimen. Corticosteroids were also frequently used for treatment of SARS-CoV and MERS-CoV infection because these viruses also induce an increased level of cytokines. According to WHO interim guidance, systemic corticosteroids should not be routinely given, because previous studies in patients with SARS-CoV and MERS-CoV suggest that use of corticosteroids have no effect on mortality, rather it delays viral clearance (Huang et al., 2020). However, in patients with severe COVID-19 infection that are also suffering from cytokine storm syndrome, this hyperinflammatory condition can be reduced by the use of steroids that leads to immunosuppression in the patient. The hyperinflammatory condition can also be reduced by cytokine blockade drugs like anakinra (IL-1 blockade) and tocilizumab (IL-6 receptor blockade). A randomized control trial of anakinra has shown benefits in patients with sepsis without posing further adverse effects (Mehta et al., 2020). Novel therapy approaches that targets IL-1, IL-6, IL-18, and IFN $\gamma$ are also available. Currently, the use of Janus kinase (JAK) inhibition to dampen inflammatory cytokine signaling is also being reported to treat CSS. JAKs are a family of nonreceptor proteins that interact with cytokine and other transmembrane receptors to enhance the inflammatory process and hence exacerbating the cytokine storm. Such JAK inhibition lessens the effect of these JAK proteins and decreases the burden of COVID-19. The US Food and Drug Administration (FDA) approved an anti-IFN $\gamma$ antibody which is currently being tested to treat secondary forms of CSS (Wang et al., 2020). Further studies are needed to assess the effects of systemic corticosteroids in patients infected with SARS-CoV-2.

The current anti-viral drugs being used are remdesivir, oseltamivir, ganciclovir, ritonavir, and lopinavir. Remdesivir was used first time in a COVID-19 pneumonia patient in the USA. Trials on the use of remdesivir against COVID-19 infection are going on (Guan et al., 2020). There is preclinical evidence that remdesivir is a potent effective drug for the treatment of SARS-CoV and MERS-CoV infections. A combination of ritonavir and lopinavir provided clinical benefits in SARS-CoV infected patients, therefore clinical trials have also been started to assess the efficacy of this combination (Chen et al., 2020). Also, lopinavir combined regimen is proved to have a positive impact on patients infected with SARS-CoV-2, that is evident by continuous improvements in eosinophil levels, radiologic findings, and viral load among the discharged patients. It is suggested that 
increase in the eosinophil level after starting treatment in COVID-19 patients, is a sign of recovery in the patients (Liu et al., 2020).

Either a single antibiotic or a combination of antibiotics, covering common or some atypical pathogens can be administered in patients. Additionally, antibiotics and antifungal drugs are also being administered in case of secondary bacterial and fungal infections. The most commonly used antibiotics are carbapenems, quinolones, cephalosporins, linezolid, and tigecycline (Huang et al., 2020).

\section{CONCLUSION}

SARS-CoV-2 is transmitting rapidly from human to human contact and currently, there is no proper treatment for the disease. The clinical features range from fever, cough, headache to less common gastrointestinal symptoms i.e. diarrhea and vomiting. Mortality risk is greater in patients with acute respiratory distress syndrome and older patients with comorbidities. There is a dire need to thoroughly study the pathophysiology, differential diagnosis on the basis of clinical manifestations of the patients, and to find an effective treatment for COVID-19.

\section{REFERENCES}

Chen, N., Zhou, M., Dong, X., Qu, J., Gong, F., Han, Y., ... Yu, T. (2020) Epidemiological and clinical characteristics of 99 cases of 2019 novel coronavirus pneumonia in Wuhan, China: a descriptive study. The Lancet, 395(10223), 507-513. https://doi.org/10.1016/S01406736(20)30211-7

Guan, W., Ni, Z., Hu, Y., Liang, W., Ou, C., He, J., ... Zhong, N. (2020). Clinical characteristics of 2019 novel coronavirus infection in China. MedRxiv. https://doi.org/10.1101/2020.02.06.20020974

Huang, C., Wang, Y., Li, X., Ren, L., Zhao, J., Hu, Y., ... Gu, X. (2020). Clinical features of patients infected with 2019 novel coronavirus in Wuhan, China. The Lancet, 395(10223), 497-506. https://doi.org/10.1016/S0140-6736(20)30183-5

ICTV. (2018). Virus Taxonomy: 2018 Release ICTV.

Liu, F., Xu, A., Zhang, Y., Xuan, W., Yan, T., Pan, K., ... Zhang, J. (2020). Patients of COVID-19 may benefit from sustained lopinavir-combined regimen. International Journal of Infectious Diseases, 4019. https://doi.org/10.1016/j.ijid.2020.03.013

Mehta, P., McAuley, D. F., Brown, M., Sanchez, E., Tattersall, R. S., \& Manson, J. J. (2020). COVID-19: consider cytokine storm syndromes and immunosuppression. The Lancet, 6736(20), 19-20. https://doi.org/10.1016/ S0140-6736(20)30628-0

Tisoncik, J. R., Korth, M. J., Simmons, C. P., Farrar, J., Martin, T. R., \& Katze, M. G. (2012). Into the Eye of the Cytokine Storm. 16-32. https://doi.org/10.1128/MMBR.05015-11

Wang, D., Bo, H., Chang, H. (2020). Clinical Characteristics of 138 Hospitalized Patients With 2019 Novel Coronavirus-Infected Pneumonia in Wuhan, China. JAMA, 323(11), 1061-1069. https://doi.org/ 10.1001/jama.2020.1585

Xiaohua, C., Binhong, Z., Yueming, Q., Yurou, C., Jie, X., Yong, F., ...Feng, L. Detectable serum SARS-CoV-2 viral load (RNAaemia) is closely associated with drastically elevated interleukin 6 (IL-6) level in critically ill COVID-19 patients. MedRxiv. https://doi.org/10.1101/2020.02.29.20029520

Yang, X., Yu, Y., Xu, J., Shu, H., Xia, J., Liu, H., ... Wang, Y. (2020) Clinical course and outcomes of critically ill patients with SARS-CoV2 pneumonia in Wuhan, China: a single-centered, retrospective, observational study. The Lancet 2600(20), 1-7. https://doi.org/10.1016/S2213-2600(20)30079-5 